\title{
La administración de lo urgente y lo importante en la empresa moderna ${ }^{1}$ The administration of the urgent and the important in the modern company
}

\author{
Samuel Antonio Vargas Vargas ${ }^{2}$ \\ saanva2@gmail.com
}

\section{Resumen}

El ambiente empresarial producto de la actual dinámica de vida, exige esfuerzo y dedicación para alcanzar las metas propuestas. Estas actividades son urgentes e importantes y en muchas ocasiones se realizan las urgentes y se dejan de lado las importantes; dado que estas últimas nunca van a llegar a tener el mismo sentido de urgencia que el trabajo diario.

Este escrito muestra la diferencia entre lo urgente y lo importante en la empresa y cómo el saber diferenciar ambos conceptos ejercerá una influencia positiva en los resultados de una organización. El desafío radica en lograr que en el día a día se ejecute lo primordial, para lo cual se requiere disciplina, control, y de estrategias que permitan invertir el tiempo justo en las tareas que redunden en el éxito empresarial.

Palabras clave: Urgente; Importante; Administración del tiempo; Gerencia estratégica.

\begin{abstract}
Business environment, product of the current dynamic of life, requires effort and dedication to achieve the proposed goals. These activities are urgent and important, and in many occasions the urgent ones, are carried out while the important ones are left out; since the latter will never have the same sense of urgency as daily work.

This paper shows the difference between the urgent and the important in the company and how knowing how to differentiate both concepts will have a positive influence on the results of an organization. The challenge lies in achieving that the paramount thing is executed in the day to day, for which discipline, control, and strategies that allow to invest the right time in the tasks that result in business success are required.
\end{abstract}

Keywords: Urgent; Important; Time management; Strategic management.

\footnotetext{
${ }^{1}$ Artículo de reflexión.

${ }^{2}$ Especialista en Alta Gerencia en Mercadotecnia y Aprendizaje Autónomo. Docente de la Universidad Pedagógica y Tecnológica de Colombia - UPTC.

ORCID https://orcid.org/0000-0002-5272-5041

${ }^{*}$ Cómo citar este artículo: Vargas, S. (2018). La administración de lo urgente y lo importante en la empresa moderna. Hojas y Hablas, (15), 133-142. DOI: 10.29151/hojasyhablasn.15a8
} 


\section{Introducción}

¿Qué se entiende por gestión del tiempo? Claessens, Van Erde, Rutte y Roe (2007) consideran la gestión del tiempo como el proceso por el cual un individuo logra realizar más eficazmente las tareas y metas, un proceso por el cual una persona obtiene el control del momento y del contenido de su actividad. Así mismo Aponte y Pujol (2012) establecen que la gestión o administración del tiempo, es un proceso en el que se establecen metas u objetivos claros, se determinan las herramientas que favorecen la gestión del tiempo. En cualquier caso, la gestión del tiempo se relaciona con el trabajo en el uso del tiempo para realizar las tareas.

Cabe considerar, que los cambios vertiginosos en la economía mundial provocados por la globalización, la competitividad y la tecnología, han llevado a que las empresas consideren nuevas formas de organización y gestión, que implican cambios en la cultura organizacional. Con los cambios mencionados, las actividades empresariales son cada vez más dinámicas y requieren de muchos esfuerzos para lograr los objetivos trazados. No obstante, todos los días nos encontramos más que nunca, como prisioneros de lo urgente.

Basta revisar las actividades cotidianas en la empresa para evidenciar que se encuentran inmersos en una serie de reuniones, algunas de ellas estériles, infinidad de informes, de mensajes en el correo electrónico, en el teléfono móvil, quejas de los clientes y pedidos imposibles e interrupciones, entre otros. Estas situaciones impiden realizar las acciones que tienen que ver con el alcance de los objetivos empresariales.

La queja más común de los directivos y profesionales a todos los niveles es que el tiempo del que se dispone no es suficiente para hacer todo lo que desea hacer. Básicamente se dedica gran parte de las actividades de la empresa a trabajar bajo uno o más formas de actuar, dependiendo de cómo se clasifiquen esas tareas bien sea como urgentes o como importantes. No se sabe a ciencia cierta ¿cuándo es urgente o importante un evento en la empresa? El saber diferenciar un asunto urgente de uno importante es de vital importancia, para que se consigan los objetivos propuestos $y$ para que la empresa sea exitosa. Por consiguiente, se planteó como objetivo identificar las diferencias entre lo urgente y lo importante, así como mostrar como lo importante se relaciona con el propósito estratégico.

La respuesta al anterior interrogante se realiza a partir del estudio de los postulados de la administración del tiempo y la planeación estratégica para hacer una reflexión de sus implicaciones en el proceso de construir un futuro promisorio para la organización.

\section{Metodología}

La metodología fue desarrollada en el contexto de las ciencias sociales y administrativas, haciendo énfasis en el pensamiento administrativo y se revisaron las teorías organizacionales. El estudio se desarrolló con un enfoque hermenéutico que permitió el análisis de textos y la interpretación de la información con métodos bibliográficos que accedieron a la captación plena del sentido de los textos consultados, tal como lo menciona Ricoeur (1984, p. 12) "Interpretar una obra es descubrir el mundo al que ella se refiere en virtud de su disposición, de su género y de su estilo".

El método utilizado fue el documental, consistente en un análisis de la información escrita sobre el tema en cuestión, donde se observa y se 
reflexiona sistemáticamente sobre realidades, con el propósito de establecer relaciones o diferencias. Para ello la investigación documental recorre varias etapas: la selección del tema, la búsqueda y selección de fuentes, la interpretación de la informaciónylasconclusiones. Los procedimientos se apoyaron en el análisis de diferentes tipos de documentos, combinando materiales de trabajo con la experiencia del investigador para, de esta forma, establecer las conclusiones.

\section{Análisis, resultados}

El análisis se da observando la organización del trabajo y el empleo del tiempo para conocer con exactitud si la empresa sufre de alteraciones en estos aspectos, Mengual, Juárez, Sempere y Rodríguez (2012) mencionan que es a través de la identificación y/o manifestación de los siguientes síntomas que se determina la gestión eficaz del tiempo.

- Jornadas cada vez más largas de trabajo, no saber decir "no", el perfeccionismo, y no negociar plazos de respuesta.

- Falta de un plan de trabajo diario o considerar los imprevistos o utilizar mal las comunicaciones (teléfono, fax, correo, mensajeros, visitas).

- Abordar las tareas en tiempo real o hacerlas de prisa o uno mismo, no delegar, así como el estrés, y el cansancio constante.

Muchos de los empleados tienen la convicción de que son los demás los causantes de esta problemática. Pero esto no es del todo cierto, puesto que cuando se examina como se utiliza el tiempo, los resultados muestran que son los propios hábitos los que le permiten o no un aprovechamiento adecuado de este recurso llamado tiempo, cuyas manifestaciones se traducen en que se realizan ciertas tareas y otras se aplazan.

\section{¿Por qué realizamos ciertas tareas y no otras?}

Por razones simples, el ser humano tiene la tendencia de anteponer ciertas actividades y dejar otras para después; un después que fácilmente se convierte en nunca; a título ilustrativo, se indican las que se llevan a cabo:

- Son agradables, son cortas y requieren poco tiempo para su realización, son fáciles de ejecutar. son conocidas dada su repetición.

- Son urgentes en su entrega, son interesantes en la temática, son programadas con anticipación.

- Son las que nos ordena el jefe.

- Son las que se realizan a manera de colaboración para compañeros que se aprecian.

Para dejar de postergar las tareas es fundamental "convencer a los colaboradores, que los beneficios de empezar a trabajar inmediatamente compensan con creces los inconvenientes" (Webb, 2016, párr.2).

Se retrata la importancia que tiene la gestión del tiempo para ser más eficiente y eficaces en el puesto de trabajo. Para Drucker (1967) la gestión del tiempo es una herramienta de dirección que permite que las empresas valoren cada vez más dicha gestión, ya que mejora la productividad y la competitividad de la organización.

\section{Lo urgente y lo importante en la empresa}

Dentro de esta perspectiva, es preciso distinguir lo importante y lo urgente de todo aquello que no lo es; una forma práctica para establecer dichas diferencias consiste en formular el siguiente interrogante, ¿cuándo una actividad es importante o urgente en la empresa? Para dar respuesta es necesario considerar los conceptos de urgente e importante. 
-Lo urgente. Lo constituyen todas aquellas actividades que requieren atención inmediata y son las que ponen a prueba la capacidad de respuesta momentánea. Son las que requieren una acción inmediata. ¡A Ahora! ¡Ya! ¡En este momento! Las consecuencias de no realizarlas son a corto plazo; por ello es necesario valorar la urgencia; para esto es ineludible formular una pregunta como la siguiente: ¿Qué puede ocurrir si no lo hago ahora? Si la respuesta es nada, no es urgente. A estas tareas se reacciona y punto.(Formatalent (s.f.) "diferencias entre urgente e importante", párr. $3,4)$.

-Lo importante. Son las actividades que llevan a cumplir el objetivo final de la empresa. Pueden ser a corto, mediano o largo plazo y ponen a prueba la capacidad de respuesta futura frente a los obstáculos que se van presentando en el presente. Son actividades que efectúan un aporte positivo a la misión, valores y metas de alta prioridad. Es recomendable saber identificar lo importante. Para ello se formula una pregunta como la siguiente: ¿Me ayudará esta tarea a alcanzar las metas y objetivos propuestos?

-Si la respuesta es No, no es importante -Si la respuesta es SÍ, es importante.

Para las tareas identificadas como importantes se requiere iniciativa y pro-actividad, esto significa hacer que las cosas ocurran, es pasar a la acción y romper con la zona de confort. Si no se actúa dando prioridad a lo importante es imposible que la empresa sea próspera y crezca.

\section{¿Qué se puede hacer al respecto? y ¿cómo proceder?}

A partir de estos conceptos se desarrolla la matriz de la administración del tiempo. El aprender a cuidar y a defender el tiempo en la empresa permite organizar cada aspecto de la vida empresarial. Para saber cómo se está utilizando el tiempo de forma conveniente, se expondrá a continuación la matriz de Covey (1989) en la cual se combina las dimensiones de lo importante y no importante con las dimensiones de lo urgente y no urgente. Tal como se presenta en el siguiente cuadro.

\section{Tabla 1 Matriz de administración del tiempo}

\begin{tabular}{|c|c|c|}
\hline & Urgente & No Urgente \\
\hline $\begin{array}{c}\text { (I) } \\
\text { Importante }\end{array}$ & $\begin{array}{c}\text { ¡HÁGALO YA! } \\
\text { Crisis. Quejas de clientes. } \\
\text { Problemas apremiantes. } \\
\text { Proyectos a punto de vencer. } \\
\text { Trabajos urgentes y que } \\
\text { deben entregarse. } \\
\text { Juntas o citas. Reportes. } \\
\text { Reparaciones. }\end{array}$ & $\begin{array}{c}\text { PLANEAR } \\
\text { Prevención. Construcción } \\
\text { de relaciones. } \\
\text { Reconocer oportunidades. } \\
\text { Planificación estratégica. } \\
\text { Nuevas estrategias. } \\
\text { Nuevos negocios. } \\
\text { Investigación y desarrollo. } \\
\text { La familia. }\end{array}$ \\
\hline $\begin{array}{c}\text { (II) } \\
\text { No } \\
\text { Importante }\end{array}$ & $\begin{array}{l}\text { RECHAZAR, DELEGAR } \\
\text { Interrupciones. Llamadas. } \\
\text { Correos. Algún informe. } \\
\text { Algunas reuniones. } \\
\text { Cuestiones inmediatas. } \\
\text { Actividades populares. } \\
\text { Caprichos del jefe que no } \\
\text { tienen que ver con el trabajo. }\end{array}$ & $\begin{array}{c}\text { DESECHAR } \\
\text { NO LO HAGA } \\
\text { Navegar por internet. } \\
\text { Trivialidades. Ajetreo } \\
\text { inútil. } \\
\text { Chismes. Algunas } \\
\text { llamadas. } \\
\text { Pérdidas de tiempo. } \\
\text { Actividades de escape } \\
\text { Messenger, WhatsApp. }\end{array}$ \\
\hline
\end{tabular}

Fuente: Covey (1989). 
Con base en lo expuesto y para efectos del presente artículo, solo se tomarán en cuenta, los cuadrantes relacionados con el SI, los cuadrantes I, y II.

\section{-Cuadrante I. Acciones urgentes e importantes.}

Tal como se ha mencionado son actividades que se deben realizar de inmediato, dado que son tareas de alta prioridad. Por consiguiente, serán situaciones de agotamiento, y quizás deba gastarse dinero extra para solucionar el problema. La empresa en ninguna circunstancia debe estar orientada hacia a este rango de acción, no puede permitirse apagar fuegos todos los días pues no sería rentable.

-Cuadrante II. Acciones no urgentes e importantes. Son aquellas actividades que SÍ significan un avance en la empresa o negocio. Por ello estas acciones harán que la compañía evolucione de forma positiva y constante. Todas aquellas empresas que gran parte de su actividad está centrada en la gestión de este tipo de acciones son las que realmente prosperan.

Acerca de la administración del tiempo, en la literatura predomina la concepción de la gestión del tiempo del directivo como una competencia del liderazgo. En este sentido, se destaca la aportación de Hellsten (2012) quien afirma "que la gestión del tiempo es una competencia que incluye, entre otras cosas, establecer y respetar prioridades, para ser más eficiente realizando las tareas y alcanzando sus objetivos".

\section{¿Qué debe predominar en la empresa?}

Según lo planteado en los cuadrantes lo que predomina en la empresa es lo importante, es decir lo estratégico, representado en la matriz de Covey en el cuadrante II cuando hace relación a planeación estratégica y nuevas estrategias, decisiones que tienen que considerar lo estratégico, entendiéndose como el cumplimiento de la misión y visión empresarial, aspectos claves que trazan el direccionamiento hacia la consecución de los objetivos. Así las cosas, resulta claro que se requiere destinar el tiempo necesario dado que se trata de llevar a cabo las acciones que deben iniciarse en el presente para realizar la prospectiva futura de la organización. Significa que se debe ir reduciendo aquellas tareas urgentes, y de esta manera el quehacer corporativo esté lleno de actividades no urgentes, pero si importantes.

\section{Lo importante, lo estratégico en la empresa}

Para lograr que la empresa realice siempre lo importante (lo estratégico) hará uso de una herramienta administrativa muy popular entre la Gerencia y esto es la Gestión estratégica, que recoge los postulados de la Planeación Estratégica y se enfoca en la implantación de la estrategia en la organización. No obstante, en la cotidianidad empresarial, gran parte de los procesos de planeación estratégica se conceptualizan y se ejecutan en forma deficiente, a menudo, el proceso no es muy creativo y es de naturaleza táctica en vez de estratégica (Goodstein, 1998, p.442). Sin embargo, antes de profundizar en este tema, se considera importante plasmar algunas precisiones sobre el concepto de estratégico, y para ello se hace alusión a una Ley de Murphy, poco conocida, la ley de la trivialidad, la cual plantea "que el esfuerzo dedicado a una decisión es inversamente proporcional a su importancia" (Murphy, 2013). De acuerdo con esta ley, las decisiones importantes, reciben menos atención que aquellas decisiones más triviales. Es necesario entonces que la Dirección de la empresa y sus ejecutivos desafíen esta ley y le asignen su limitado tiempo de manera que las decisiones más importantes, 
(las estratégicas), reciban relativamente mayor atención.

Con base en lo planteado, surge la inquietud, de cómo identificar las decisiones estratégicas (importantes) en la empresa. Debe señalarse que las decisiones estratégicas no son rutinarias, son complejas, por cuanto comprometen una gran cantidad de recursos económicos, financieros y humanos, que determinan el futuro de la compañía a mediano y largo plazo. Son responsabilidad de la alta gerencia, que las toma luego de realizar un análisis pormenorizado de las condiciones del mercado. De este modo, son estas razones las que determinan la importancia de lo estratégico. Visto de esta forma, para identificar las decisiones estratégicas es imprescindible considerar un criterio práctico: Las decisiones estratégicas son aquellas más irreversibles, las que determinan un mayor compromiso, como lo afirma Melara (2013).

En esta misma forma Mintzberg (1997), relaciona que "las decisiones estratégicas son aquellas que establecen la orientación general de la empresa y su viabilidad maximiza a la luz, tanto de los cambios predecibles como impredecibles" ( $p$. 641). De lo anterior se deduce que en la compañía puede encontrarse con dos tipos de decisiones: las primeras basadas en estrategias que son reversibles cada una de impacto relativamente menor y las segundas implican compromisos irreversibles. Todas las decisiones deben ser analizadas considerando su repercusión en la estructura de la industria, en las ventajas competitivas en costo y en diferenciación. Mientras que las decisiones de carácter irreversible, requieren de un análisis más profundo y cuantitativo, que incluye usar el método deductivo; el cual consiste en evaluarlas utilizando los principios económicos y de gestión para estudiar las alternativas y elegir racionalmente. (Del Sol, 2009).

\section{Lo verdaderamente importante}

Para definir lo estratégico (lo importante) la empresa tomará como herramienta la Gerencia y la planeación estratégica, para facilitar el desarrollo del logro de los objetivos organizacionales, y estructurar el norte estratégico, esto es a donde se quiere llegar en el largo plazo y orientar las estrategias de crecimiento y competitividad, así mismo se convierte en un elemento de confianza hacia los stakeholders (Hitt, 2001).

-Concepto de estrategia. La concepción de estrategia se aplica en la Administración Estratégica cuando se refiere a organizaciones empresariales, Andrews (como se citó en Vargas, 2014) en el sentido de adaptar conjuntamente recursos y capacidades de ellas a un entorno permanentementevariable, en el cual se desempeña dicha organización. Por su parte Ohmae (2004,p. 16), define la estrategia como la forma en que una corporación se comporta para diferenciarse a sí misma positivamente frente a sus competidores usando sus fortalezas relativas con el propósito de satisfacer mejor las necesidades de los clientes. En tanto que para Porter (1980, p.35), la estrategia competitiva consiste en desarrollar una amplia formula de cómo la empresa va a competir, cuáles deben ser sus objetivos y qué políticas serán necesarias para alcanzar tales objetivos.

Es la dinámica de la relación de la empresa con su entorno para el que se toman las acciones necesarias de cara a alcanzar sus objetivos y/o para aumentar el rendimiento mediante el uso racional de los recursos. No obstante, del análisis de distintas definiciones de estrategia se destaca que la estrategia se apoya en cuatro elementos (Andrews. 1977):

- La estrategia es una relación entre la empresa y su entorno. 
- Es una respuesta a las expectativas tanto del empresario como las del cliente.

- Es un modelo de decisión para cumplir los objetivos.

- Es un sistema para la solución de problemas empresariales.

Por su parte Steiner (1994, p.20) propone que la Planeación Estratégica es un el elemento integrador entre el diseño de una estrategia, su implementación y la participación de los actores en distintos niveles. Siendo las cosas así resulta claro que toda estrategia empresarial tiene un propósito.

\section{Propósito de la Dirección Estrategia}

La Dirección Estratégica define el futuro de una organización, por cuanto le permite constituirse en una empresa de carácter proactiva y no reactiva. El propósito radica en posibilitar el cambio, dada las siguientes razones:

- Definir con exactitud cuál es el negocio en el que se encuentra inmersa la Organización (misión de la empresa). A dónde quiere ir (visión).

- Determinar los objetivos empresariales, tanto a corto, mediano y largo plazo.

- Establecer las estrategias más adecuadas para el negocio.

- Incrementar en los administradores el pensamiento estratégico.

- Lograr la competitividad estratégica y rendimientos superiores al promedio.

Cuando una empresa pone en práctica una estrategia que crea valor y de la cual, otras compañías no pueden duplicar los beneficios o la consideran demasiado costosa para imitar (Barney, 1996), entonces esta empresa cuenta con una ventaja sostenida o sostenible (ventaja competitiva).
Uno de los grandes retos de la administración es cómo obtener rendimientos superiores, objetivo que por sí solo es una decisión supremamente importante, y una vez fijado hay que apegarse al direccionamiento estratégico el cual representa un conjunto de acciones y de decisiones, que se requieren para que la empresa logre la competitividad estratégica y alcance rendimientos superiores al promedio. Por ello es fundamental contar con la información de carácter estratégico relevante, la cual proviene de los análisis de los ambientes interno y externos y que es prioritaria para elaborar una estrategia y una implementación efectiva de la misma.

Las acciones estratégicas de efectividad son un requisito indispensable para lograr los resultados deseados (competitividad estratégica y rendimientos superiores al promedio). Estas acciones estratégicas tienen lugar en el contexto de los procesos de formulación e implantación de la estrategia que cuidadosamente integrados dan como resultado los resultados estratégicos deseados.

En esta concepción, existen dos modelos que sugieren algunas condiciones que la empresa debe analizar a fin de obtener la información estratégica necesaria para seleccionar las acciones efectivas y alcanzar los resultados. Ambos referentes hacen hincapié en aspectos diferentes. El primero denominado de la Organización Industrial, señala que el ambiente externo es el factor determinante de las gestiones estratégicas de una compañía; la clave está en la ubicación y competencia exitosa en una industria atractiva (modelo de las 5 fuerzas de Porter). El segundo designado con base en los recursos, sugiere que los recursos y las capacidades (análisis interno) son lo esencial en determinar las tareas estratégicas exitosas. 
No se trata en pensar únicamente en la rentabilidad, se trata también de pensar en la satisfacción de las necesidades del cliente. La empresa debe proveer los beneficios y satisfactores que el cliente desea a la hora de comprar un bien o servicio. Satisfacer las necesidades de los clientes obedece a una premisa importante para la empresa, porque los rendimientos al final del ejercicio contable se obtienen de las relaciones con ellos, convirtiéndose en el elemento vital de toda organización. En resumidas cuentas, las empresas han comprendido que no se puede atraer a todos los compradores del mercado, han entendido que son demasiados y que se encuentran dispersos con prácticas de compra de diversa índole. (Vargas, 2012). En consecuencia, utilizan sus recursos y capacidades para formular estrategias a fin de crear valor y satisfacer las necesidades de los clientes.

En el marco teórico se ha destacado la importancia de la gestión del tiempo en relación con lo urgente y lo importante, en el sentido que lo verdaderamente transcendental para la empresa recae sobre el direccionamiento estratégico. Camacho (2002 afirma que "el direccionamiento estratégico es un enfoque gerencial que permite a la alta dirección determinar un rumbo claro, y promover las actividades necesarias para que toda la organización trabaje en la misma dirección" ( $p$, 7). Por ello una buena administración del tiempo se considera como una competencia cada vez más significativa para la planeación, la organización, la dirección y el control de los objetivos planteados.

Lo urgente son actividades que se deben realizar de inmediato, son tareas que presionan a diario y que requieren de una respuesta inmediata. En nuestra opinión son asuntos urgentes:

- Crisis al interior de la empresa, quejas de clientes por el producto o servicio, problemas apremiantes como compra o mantenimiento de equipos.

- Proyectos a punto de vencer y que deben entregarse.

- Juntas, citas del personal o clientes por atender.

- Reportes o informes relacionados con la gestión.

Así mismo, las actividades importantes son aquellas que significan el sostenimiento y competitividad en el mercado, las cuales requieren del tiempo preciso para su ejecución. Se logran haciendo uso de la planeación y la gerencia estratégica. En este sentido se señala como acciones importantes:

- Captación y fidelización de clientes.

- Construcción de relaciones estratégicas con otros negocios, mediante alianzas, o convenios.

- Diseño de planes estratégicos.

- Nuevas estrategias de negocio, o corporativas

- Diseño de estrategias de marketing,

- Investigación y desarrollo.

- Capacitación al personal.

Igualmente se ha aludido a la utilización de determinadas herramientas de gestión del tiempo como la matriz de Covey, señalándose como un instrumento valioso para mejorar la productividad mediante la priorización de las tareas. Así mismo la matriz, en el cuadrante II, relaciona la planificación y la estrategia como decisiones importantes. En este marco se comprende puesto que la gerencia estratégica (planeación, estrategia) le permite a una organización aumentar la eficiencia, satisfacer las necesidades de los clientes, y ser proactiva en vez de reactiva en la formulación de su futuro. De hecho, la formulación y ejecución de la estrategia es la que orienta la consecución de los objetivos propuestos en toda organización. 


\section{Conclusiones}

Se considera que:

El saber diferenciar lo urgente e importante en la empresa es de vital importancia para no distraerse en actividades que impidan alcanzar los objetivos empresariales, es decir, que una adecuada gestión del tiempo por parte de los directivos produce efectos positivos en su trabajo, en el trabajo de los demás miembros de la empresa y en los resultados.

Si bien en la organización se presenta a diario un sinnúmero de actividades, es necesario comprender que las tareas importantes se les debe asignar el tiempo justo para su realización, dado que tienen que ver el direccionamiento estratégico, y en la consecución de los objetivos empresariales en el corto, mediano y largo plazo.

La administración y gestión del tiempo en la empresa es esencialmente un esfuerzo de equipo $y$ se constituye en una competencia gerencial que requiere de un liderazgo efectivo e implica coordinación de actividades, ayuda mutua y cooperación por parte de los miembros de la organización.

Es de gran relevancia que el tiempo en la empresa sea visto como un recurso valioso que no se debe desaprovechar, por ello debe tenerse claro cuáles son las prioridades más altas, para de esta manera decir no, a otras actividades que no permiten la realización precisa del propósito estratégico de la empresa la misión y la visión de la misma.

Relacionar lo importante con lo estratégico, permite entonces considerar que, en las organizaciones, no importa su tamaño, se debe tomar decisiones que en algunos casos son reversibles y en contadas ocasiones de carácter irreversibles, las cuales requieren de una atención especial dado que implica todo el accionar estratégico de la empresa, por lo que se requiere administrar y gestionar efectivamente el tiempo para su correcta toma y aplicación.

\section{Referencias}

Aponte, E. D. y Pujol, L. (2012). Estilos de aprendizaje, gestión del tiempo y rendimiento académico en estudiantes universitarios. En: Estilos de aprendizaje: investigaciones y experiencias. V Congreso Mundial de Estilos de Aprendizaje, Santander, 27, 28 y 29 de junio de 2012.

Andrews, K. (1977). El concepto de estrategia de la empresa. Pamplona: Universidad de Navarra.

Barney, J. (1986). Strategic factor markets: Expectations, luck, and business strategy. Management Science, 32(10), 1231-124l.

Camacho, M. (2002). Direccionamiento estratégico: análisis de una herramienta poderosa. Revista Vía

Salud, (21), 6-12. Recuperado de https://goo.gl/ F2QP3Z

Claessens, B., Van Erde, W., Rutte, C., \& Roe, R. (2007). A review of the time management literature.

Personnel Review, 36(2), 255-276.

Covey, S. (1989). 7 hábitos para gente altamente efectiva. Buenos Aires: Paidós.

Del Sol, P. (2009). Decisiones estratégicas. Recuperado de http://www.patriciodelsol.com/curriculum/

Drucker, P. (1967). El ejecutivo eficaz. Sudamericana. Bogotá. Recuperado de https://goo.gl/HJPvvd.

Formatalent. (s.f.). Diferencias entre urgente e importante. [Mensaje en un blog]. Recuperado de https://goo.gl/xm7amZ.

Goodstein, L., Timothy, M y Pfeiffer, W. (1998). Planeación estratégica aplicada. Bogotá: McGrawHill.

Hellsten, L. (2012). What do we know about time ma- 
nagement? Review of the Literature and Psychometric Critique of Instruments Assessing. En: Todos Stoilov. (Eds), Time Management. (pp. 1-27).

Hitt, M. (2001). Administración estratégica: Competitividad y conceptos de globalización. México: Thomson.

Melara, S. M. (12 de julio de 2013). Decisiones estratégicas y no estratégicas en las empresas [Mensaje en un blog]. Recuperado de https://marlonmelara.com/decisiones-estrategicas-no-estrategicas/

Mengual, A., Juárez, D., Sempere, M. y Rodríguez, A. (2012). La gestión del tiempo como habilidad directiva. 3C Empresa, Investigación y Pensamiento Crítico, (7), 6-30.

Mintzberg, H., Quinn, J., \& Voyer, J. (1997). El proceso estratégico: conceptos, contextos y casos. México: Prentice-Hall.

Murphy, E. (10 de julio de 2013). Ley de Murphy. [Mensaje en un blog]. Recuperado en https://goo. $\mathrm{gl} / \mathrm{dnEKXM}$
Ohmae, K. (2004). La mente del Estratega. El arte de Japón en el mundo de los negocios. México: McGraw-Hill.

Porter, M. (1992). Ventaja competitiva. Creación y sostenimiento de un desempeño superior. México: CECSA.

Ricoeur, P. (1984). La metáfora viva. Buenos Aires: Megápolis

Steiner, G. (1994). La planeación estratégica. Lo que todo director debe saber, México: CECSA.

Vargas, S. (2012). Marketing agropecuario. México: Trillas. Recuperado de https://goo.gl/swaV9Q

Vargas, S (2014) Como implementar un proceso de dirección estratégica. Recuperado de https://goo. gl/95MTC3

Webb, C. (2016). How to Beat Procrastination. Harvard Bussines Review. Recuperado de https://goo. gl/2Jgcc8

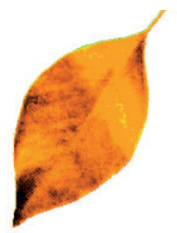

